\title{
Caries preventive efficacy of silver diammine fluoride (SDF) and ART sealants in a school-based daily fluoride toothbrushing program in the Philippines
}

Bella Monse ${ }^{1 *}$, Roswitha Heinrich-Weltzien ${ }^{2}$, Jan Mulder ${ }^{3}$, Christopher Holmgren ${ }^{4}$ and Wim $\mathrm{H}$ van Palenstein Helderman ${ }^{5}$

\begin{abstract}
Background: Occlusal surfaces of erupting and newly erupted permanent molars are particularly susceptible to caries.

The objective of the study was to assess and compare the effect of a single application of 38\% SDF with ART sealants and no treatment in preventing dentinal (D3) caries lesions on occlusal surfaces of permanent first molars of school children who participated in a daily school-based toothbrushing program with fluoride toothpaste.

Methods: The prospective community clinical trial in the Philippines was conducted over a period of 18 months and included 704 six- to eight-year-old school children in eight public elementary schools with a daily school-based fluoride toothpaste brushing program. Children were randomly assigned for SDF application or ART sealant treatment. Children from two of the eight schools did not receive SDF or ART sealant treatment and served as controls. SDF or ART sealant treatment was applied on sound occlusal surfaces of permanent first molars. Surfaces that were originally defined as sound at baseline but which changed to dentinal (D3) caries lesions were defined as surfaces with new caries (caries increment). Non-compliance to the daily toothbrushing program in three schools offered the opportunity to analyze the caries preventive effect of SDF and sealants separately in fluoride toothpaste brushing and in non-toothbrushing children.
\end{abstract}

Results: In the brushing group, caries increment in the SDF treatment group was comparable with the non-treatment group but caries increment in the sealant group was lower than in the non-treatment group with a statistically significant lower hazard ratio of $0.12(0.02-0.61)$. In the non-brushing group, caries increment in the SDF treatment group and the sealant group was lower than the non-treatment group but the hazard ratio was only statistically significant for the sealant group (HR 0.33; 0.20-0.54). Caries increment was lower in toothbrushing children than in non-toothbrushing children. Hazard ratios reached statistical significance for the non-treated children (HR 0.43; 0.21-0.87) and the sealant-treated children (HR 0.15; 0.03-0.072).

Conclusions: A one-time application of $38 \%$ SDF on the occlusal surfaces of permanent first molars of six- to eight-year-old children is not an effective method to prevent dentinal (D3) caries lesions. ART sealants significantly reduced the onset of caries over a period of 18 months.

Trial registration number: German Clinical Trial Register DRKS00003427

Keywords: Silver diamine fluoride, Toothbrushing, Sealants, Permanent molars, Dental caries

\footnotetext{
* Correspondence: bella.monse@giz.de

'Deutsche Gesellschaft für Internationale Zusammenarbeit (GIZ) GmbH, GIZ Office Manila, PDCP Bank Centre, V.A. Rufino cor. L.P. Leviste Str, Makati City, Metro Manila, Philippines

Full list of author information is available at the end of the article
} 


\section{Background}

Dental caries is a global pandemic [1,2]. Treatment of caries in children is virtually non-existent in a number of low- and middle-income countries and, in children under six years of age, it is limited even in many highincome countries [3]. The situation in the Philippines, a low-middle-income country, is no different. According to the 2006 Philippine National Oral Health Survey, 97\% of the six-year-old children and $82 \%$ of the 12 -year-olds suffer from dental caries and the few restorations observed indicate that restorative treatment is rather rare [4]. In both age groups just over $40 \%$ of existing caries lesions have progressed to odontogenic infections [5]. Furthermore, $20 \%$ of 6 -year-olds and $16 \%$ of 12 -yearolds reported having a problem in their mouth at the time of being questioned [4]. According to the Department of Education, the principal reason for absenteeism from school in the Philippines is toothache [6]. Dental caries in children appears to be the most prevalent childhood disease in the Philippines followed by soiltransmitted helminthiasis [7]. Moreover, the presence of odontogenic infections in 12-year-olds appears to be associated with a low Body Mass Index (BMI) and this association might represent a significant yet largely neglected determinant of poor child development [8].

The dramatic decline in caries over the past three decades, seen in many high-income countries, is largely attributed to the widespread use of fluoride toothpaste, in spite of continued consumption of high levels of sugar $[9,10]$. There are no mass fluoridation schemes in the Philippines and the high caries experience in Filipino children suggests that the use of fluoride toothpastes with anti-caries efficacy is minimal, although no reliable data exist. This emphasizes the urgent need for appropriate exposure to fluoride in the country, which should be an essential component of any preventive oral health care program [11-14]. The Global Consultation on Oral Health through Fluoride [12], which was jointly convened by the World Health Organization (WHO), the FDI World Dental Federation (FDI) and the International Association for Dental Research (IADR) in November 2006 in Geneva stated that "Prevention by using fluoride is the only realistic way of reducing this burden (of dental caries) in populations". In addition, the WHO, in 2007, at the 60th World Health Assembly [13] urged governments "to promote oral health in schools, aiming at developing healthy lifestyles and self-care practices in children" while the declaration of the Beijing conference in 2007 [14] which was jointly convened by the WHO, the FDI, the IADR and the Chinese Stomatological Association (CSA) stated that "fluoride toothpaste remains the most widespread and significant form of fluoride used globally and the most rigorously evaluated vehicle for fluoride use".
In 2004, several years before these international recommendations were proclaimed, a pilot project was initiated in the Philippines consisting of daily schoolbased toothbrushing with fluoride toothpaste for the children of eight elementary public schools in Cagayan de Oro (Mindanao) and Manila.

It was anticipated that daily school-based toothbrushing with fluoride toothpaste, starting in grade 1 schoolchildren, would impact on the caries levels of the permanent dentition [15]. It was also hoped that a single application of $38 \%$ silver diammine fluoride (SDF) would provide additional caries preventive effect over and above that provided by the regular use of fluoride toothpaste. Any additional caries preventive effect would be particularly useful to tide over periods of high caries susceptibility e.g. during the period of eruption of first permanent molars [16]. Even though the literature indicates that any fluoride application over a period of $2-3$ years, be it a varnish, a fluoride gel or fluoride rinse results only in a modest additional caries preventive effect of about $10 \%$ when used together with daily toothbrushing with fluoride toothpaste [17], SDF applications were not included in this systematic review. One study has shown promising results with SDF on caries prevention [18], while a systematic review concluded that SDF was more effective than fluoride varnish in preventing caries [19]. The rationale for the selection of $38 \%$ SDF as an additional preventive measure was its ease and speed of application, its low cost, and its potentially high cariespreventive and caries-arresting effect $[18,19]$. Moreover, this intervention was also selected in the perspective of being a feasible component in a national school based health program. A single application of SDF was chosen because repeated applications were unlikely to be feasible in the resource-limited setting of the Philippines.

Another, potentially more costly and more time consuming approach, is the provision of sealants. Studies have shown the effectiveness of sealants to prevent occlusal caries, and in the Philippine school setting, where dental facilities are not available, ART glassionomer cement sealants applied with the finger-press technique were considered the most appropriate sealant method [20]. These sealants placed on occlusal surfaces of permanent first molars of first graders were considered a positive control for the single 38\% SDF application.

The aim of the present study therefore was to assess and compare the effect of a single application of $38 \%$ SDF with ART sealants and no treatment in preventing dentinal (D3) caries lesions on occlusal surfaces of permanent first molars of first graders of the eight public elementary schools that participated in a daily school-based toothbrushing program with fluoride toothpaste. 


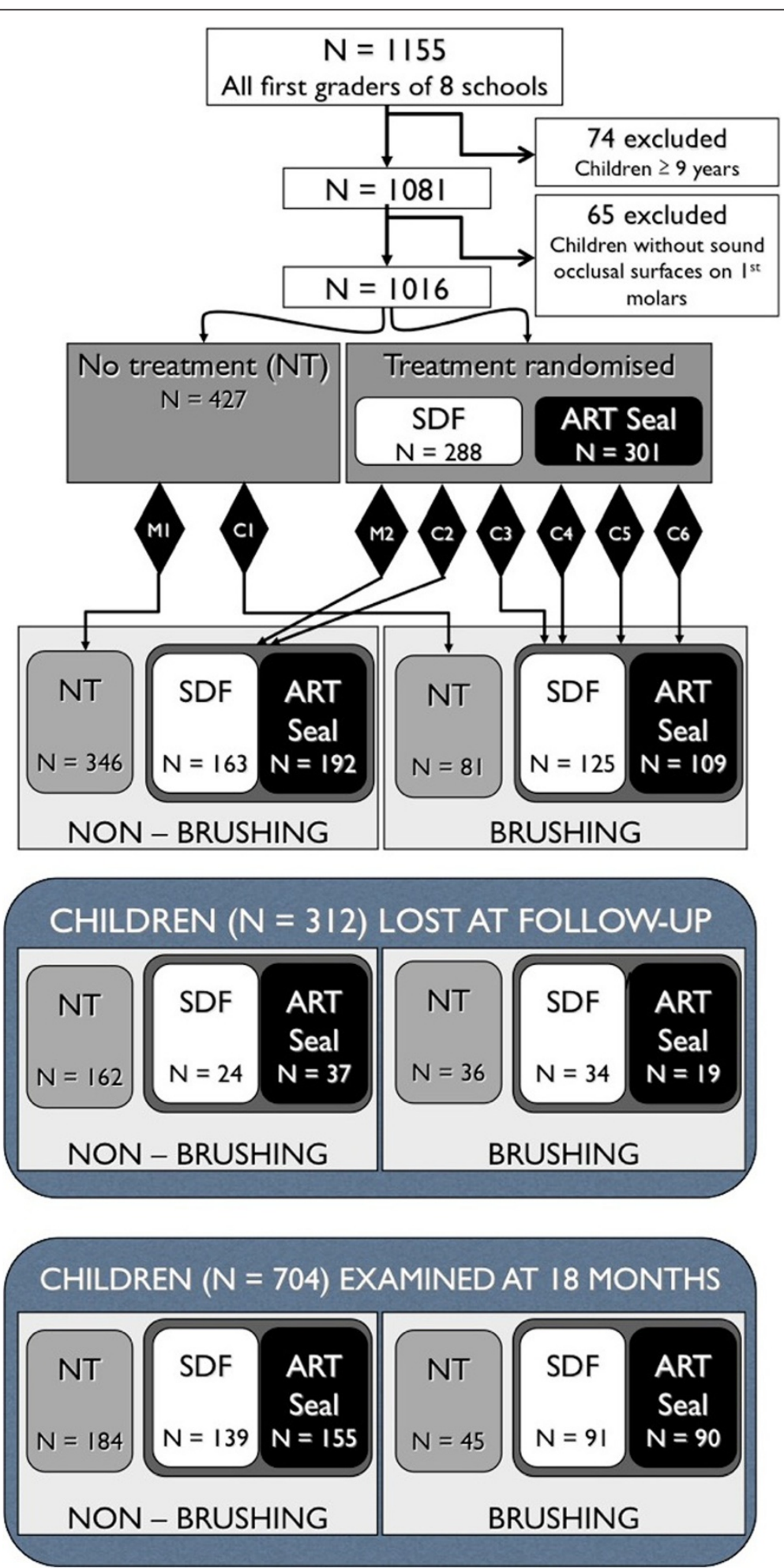

Figure 1 Flow chart of the study including participating schools, groups and students (M1 and M2 represent schools in Manila, C1 to C6 represent schools in Cagayan de Oro). 


\section{Methods}

This study, which started in 2004, was a community clinical trial on a cohort of first graders of eight public elementary schools to evaluate the effect of treatment under prevailing contextual factors of low-income countries.

\section{Study population}

Six public elementary schools were selected in Cagayan de Oro and two in Manila, in the Philippines. All eight schools were involved in an on-going oral health care program that included daily school-based toothbrushing with fluoride toothpaste (1450 ppm F) that had been implemented in the months immediately preceding the start of this study. All first graders $(n=1155)$ were examined. Inclusion criteria for the study were children of six to eight years of age in grade one who had at least one erupted permanent first molar with a sound occlusal surface. "Sound"included clinically sound and all stages of enamel caries up to but not including visible dentinal (D3) caries lesions. Of the excluded children, 74 (6.4\%) were nine years or older and 65 (5.6\%) children did not meet the inclusion criterion of having at least one erupted permanent molar with a sound occlusal surface (Figure 1). All excluded first graders $(n=139)$ received SDF treatment on decayed surfaces of the permanent dentition. Tooth extraction was offered to all first graders in cases where the caries lesion had progressed into the pulp. One of the two schools in Manila and one of the 6 schools in Cagayan de Oro were appointed by lottery as control schools (no treatment). In the remaining six schools all first graders meeting the inclusion criteria received treatment. Children with a consecutive odd number on the class register received an SDF application and those with a consecutive even number ART sealants on sound occlusal surfaces of their permanent first molars. Children assigned for SDF treatment also received an SDF application on decayed surfaces of the permanent dentition while children assigned for ART sealants received ART restorations on decayed (D3) surfaces of the permanent dentition.

Signed informed consent was obtained from the parents of all the first graders of the selected schools. The ethics commission of Xavier University, Cagayan de Oro, Philippines, approved the study protocol.

Based on an assumed 10\% reduction in caries increment in the treated group as compared to the nontreatment group, a statistical power of $80 \%$, a p-value of 0.05 and an anticipated drop out of 35\%, with 1016 included children a necessary sample size of at least 200 children per group was possible (Table 1).

\section{Examination}

The examinations were performed outside in the schoolyard with sunlight as a direct light source. The children
Table 1 Baseline caries experience on occlusal surfaces of permanent first molars per study group $(n=1016)$

\begin{tabular}{|c|c|c|c|c|c|c|}
\hline \multicolumn{7}{|c|}{ All 8 schools } \\
\hline & \multirow{2}{*}{$\begin{array}{c}\text { Children } \\
\mathrm{N}\end{array}$} & \multirow{2}{*}{$\begin{array}{l}\text { Age } \\
\text { (sd) }\end{array}$} & \multicolumn{4}{|c|}{ Surfaces N \% } \\
\hline & & & Total & Missing & Sound & D3 caries \\
\hline $\begin{array}{l}\text { Non- } \\
\text { treated } \\
\end{array}$ & 427 & $6.7(0.7)$ & 1708 & 181 10.6\% & $125773.6 \%$ & $27015.8 \%$ \\
\hline $\begin{array}{l}\text { SDF } \\
\text { treated }\end{array}$ & 288 & $6.7(0.7)$ & 1152 & $14212.3 \%$ & $88176.5 \%$ & $12911.2 \%$ \\
\hline Sealants & 301 & $6.7(0.7)$ & 1204 & $1109.1 \%$ & $95179.0 \%$ & $14311.9 \%$ \\
\hline Total & 1016 & $6.7(0.7)$ & 4064 & $43310.7 \%$ & 3089 76.0\% & $54213.3 \%$ \\
\hline
\end{tabular}

were placed in a supine position on a long classroom bench, with their heads on a pillow on the lap of the examiner, who sat behind them. Teeth were examined after drying with cotton rolls and the occlusal surface of the permanent first molars were additionally dried with cotton pellets. A CPI ball-end probe and mouth mirror were used as examination tools and caries was scored according to procedures described by WHO [21]. Sound was scored except when: 1) the CPI probe could enter a caries cavity indicating a dentinal (D3) caries lesion or 2) if the probe could not enter a small discontinuity in the enamel but a greyish appearance of the enamel was seen as a sign of undermined caries indicating a dentinal (D3) caries lesion.

\section{Calibration of examiners}

Children were examined at baseline and followed up by eight calibrated examiners. A WHO consultant undertook training and calibration of examiners over a threeday period at one local school that was not involved in the study. Intra-examiner reproducibility was assessed on $10 \%$ of the children during the baseline and the follow-up examination.

\section{Treatment regimens}

SDF treatment was provided by school nurses who had received a one-day training in the technique and who worked under the supervision of a dentist. Sealants were placed by dentists of the Department of Education who had received training in the provision of ART glassionomer cement sealants by the supplier of the material. All treatment was provided with chairside assistance. Prior to treatment a school nurse brushed all permanent first molars without toothpaste.

School children in the SDF group received one application of 38\% SDF solution (Saforide, Bee Brand Medical, Japan) on sound occlusal surfaces of all erupted permanent first molars ("sound" as defined previously). Molars were isolated with cotton rolls. Cotton pellets were then used to dry the occlusal surface. SDF was applied on the occlusal surface by rubbing for 1 minute with 
a Vivabrush (Ivoclar Vivadent $\mathrm{GmbH}$, Liechtenstein). Next, tannic acid (strong black tea) was applied to precipitate the SDF [22]. Excess was removed with a dry cotton pellet. Thereafter, a layer of Vaseline was applied to protect the SDF from saliva. Children were asked not to eat for one hour after treatment.

Children in the sealant group received ART glassionomer cement sealants on sound occlusal surfaces of all erupted permanent first molars. The treatment was provided according to the ART sealant application procedure [23]. A high-viscosity material (Ketac Molar Easymix, 3M ESPE, Germany) was used strictly according to the manufacturer's instructions.

Change in the study design due to non-compliance to the daily school-based toothbrushing program with fluoride toothpaste

During regular monitoring visits to the schools, following the implementation of the daily school-based toothbrushing with fluoride toothpaste program, within a month it became apparent that one school in Cagayan de Oro and two schools in Manila were not complying with the program. Since the study was already in progress, it was decided to maintain these three schools in the research program for evaluation at 18 months. The analysis of 18-month data on the effect of SDF and ART sealants was therefore divided into school children with daily school brushing with fluoride toothpaste and those without. The children in the non-compliant schools continued to receive a yearly distribution of one toothbrush and one sachet of fluoride toothpaste and a lecture on oral health as is routinely provided to all school children in the Philippines.

\section{Blinding of the examiners}

The eight examiners undertaking the evaluation were not involved in the treatment. They were informed about the presence of teeth with an ART sealant or ART restoration since they are often clinically indistinguishable. They were however blinded as to whether subjects had received SDF treatment or not, or whether the school was compliant with the daily toothbrushing program with fluoride toothpaste.

The evaluation was carried out after 18 months using the same examination setting, criteria, tools and examiners.

\section{Statistical analysis}

The data were analyzed with SAS 9.1 software. Intraexaminer reproducibility at tooth level at baseline and at follow-up examinations was calculated with Kappa statistics. The baseline data regarding percentages between the different groups were analyzed with chi-square tests. For the analysis on the development of new dentinal
(D3) caries lesions (caries increment) on sound occlusal surfaces of first permanent molars, a Cox proportional hazard model was applied [24] taking into account frailties correction for the child as a unit of analysis [25].

\section{Results}

Unweighted intra-examiner mean Kappa at baseline examination for caries was 0.90 and varied between 0.86 and 0.97 while the intra-examiner mean Kappa at follow-up examination was 0.93 (0.91-0.96).

The flow chart (Figure 1) shows the number of children at baseline $(n=1016)$ that met the inclusion criteria. None of the children had restorations in their permanent first molars. The chart also presents the number of children in the SDF, sealant and non-treatment group and the subsequent sub-division of these children into brushing and non-brushing groups. Finally the number of dropouts is presented of the six different groups and the number of children available $(n=704)$ for the 18months evaluation. Table 1 presents the number of children at baseline $(\mathrm{n}=1016)$, the age, the number and percentage of missing molars (unerupted) and sound occlusal surfaces and decayed (D3) occlusal surfaces according to study group. The only statistical significance between the three groups was seen in the nontreatment group that had a higher percentage of D3 occlusal surfaces (Chi square, $\mathrm{p}$ value $<0.02$ ). The baseline data for the dropouts (Table 2) did not differ in statistically significant ways from those who entered the study at baseline (Table 1). Table 3 presents the baseline data of children remaining in the study $(n=704)$. The caries level at baseline of children in the non-treatment group was higher than those in the SDF or ART sealant

Table 2 Baseline caries experience on occlusal surfaces of permanent first molars of children lost to follow up per study group $(n=312)$

\begin{tabular}{|c|c|c|c|c|c|c|}
\hline \multicolumn{7}{|c|}{ Brushing } \\
\hline & \multirow{2}{*}{$\begin{array}{l}\text { Children } \\
\mathrm{N}\end{array}$} & \multirow{2}{*}{$\begin{array}{l}\text { Age } \\
\text { (sd) }\end{array}$} & \multicolumn{4}{|c|}{ Surfaces N \% } \\
\hline & & & Total & Missing & Sound & D3 caries \\
\hline $\begin{array}{l}\text { Non- } \\
\text { treated }\end{array}$ & 36 & $6.6(0.7)$ & 144 & $2316.0 \%$ & $10270.8 \%$ & $1913.2 \%$ \\
\hline $\begin{array}{l}\text { SDF } \\
\text { treated }\end{array}$ & 34 & $6.7(0.8)$ & 136 & $1410.3 \%$ & 103 75.7\% & $1914.0 \%$ \\
\hline Sealants & 19 & $6.8(0.9)$ & 76 & $45.3 \%$ & $6079.0 \%$ & $1215.8 \%$ \\
\hline Total & 89 & $6.7(0.8)$ & 356 & $4111.5 \%$ & $26574.4 \%$ & $5014.0 \%$ \\
\hline \multicolumn{7}{|c|}{ Non-brushing } \\
\hline $\begin{array}{l}\text { Non- } \\
\text { treated }\end{array}$ & 162 & $6.8(0.7)$ & 648 & $528.0 \%$ & $48474.7 \%$ & $11217.3 \%$ \\
\hline $\begin{array}{l}\text { SDF } \\
\text { treated }\end{array}$ & 24 & $6.8(0.7)$ & 96 & $1010.4 \%$ & $7275.0 \%$ & $1414.6 \%$ \\
\hline Sealants & 37 & $6.8(0.7)$ & 148 & $106.8 \%$ & $12383.1 \%$ & $1510.1 \%$ \\
\hline Total & 223 & $6.8(0.7)$ & 892 & $728.1 \%$ & $67976.1 \%$ & $14115.8 \%$ \\
\hline
\end{tabular}


Table 3 Baseline caries experience on occlusal surfaces of permanent first molars of children per group who participated in the 18 months evaluation $(n=704)$

\begin{tabular}{|c|c|c|c|c|c|c|}
\hline \multicolumn{7}{|c|}{ Brushing } \\
\hline & \multirow{2}{*}{$\begin{array}{l}\text { Children } \\
\mathrm{N}\end{array}$} & \multirow{2}{*}{$\begin{array}{l}\text { Age } \\
\text { (sd) }\end{array}$} & \multicolumn{4}{|c|}{ Surfaces N \% } \\
\hline & & & Total & Missing & Sound & D3 caries \\
\hline $\begin{array}{l}\text { Non- } \\
\text { treated }\end{array}$ & 45 & $6.8(0.6)$ & 180 & $2413.3 \%$ & $12770.6 \%$ & $2916.1 \%$ \\
\hline $\begin{array}{l}\text { SDF } \\
\text { treated }\end{array}$ & 91 & $6.7(0.7)$ & 364 & $4311.8 \%$ & $28879.1 \%$ & 33 9.1\% \\
\hline Sealants & 90 & $6.7(0.6)$ & 360 & $236.4 \%$ & $30183.6 \%$ & $3610.0 \%$ \\
\hline Total & 226 & $6.7(07)$ & 904 & $9010.0 \%$ & $71679.2 \%$ & $9810.8 \%$ \\
\hline \multicolumn{7}{|c|}{ Non-brushing } \\
\hline $\begin{array}{l}\text { Non- } \\
\text { treated }\end{array}$ & 184 & $6.6(0.6)$ & 736 & $8211.1 \%$ & $54473.9 \%$ & $11014.9 \%$ \\
\hline $\begin{array}{l}\text { SDF } \\
\text { treated } \\
\end{array}$ & 139 & $6.7(0.6)$ & 556 & $7513.5 \%$ & $41875.2 \%$ & $6311.3 \%$ \\
\hline Sealants & 155 & $6.7(0.6)$ & 620 & $7311.8 \%$ & $46775.3 \%$ & $8012.9 \%$ \\
\hline Total & 478 & $6.7(0.6)$ & 1912 & $23012.0 \%$ & $142974.7 \%$ & $25313.3 \%$ \\
\hline
\end{tabular}

group, but the difference only reached statistical significance in the toothbrushing group (Chi square, $\mathrm{p}<0.02$ ).

Surfaces that were originally defined as sound at baseline but which changed to dentinal (D3) caries lesions actually indicated the caries increment since no restorations were found on these surfaces. Table 4 depicts the caries increment of the six different groups at the 18months evaluation.

In the toothbrushing group, caries increment for those who received SDF treatment was comparable to the non-treatment group but caries increment in the sealant group was substantially lower than in the non-treatment group with a statistically significant lower hazard ratio of 0.12 (0.02-0.61) (Table 5). In the non-brushing group, caries increment in the SDF treatment group and the sealant group was lower than the non-treatment group but the hazard ratio was only statistically significant for the sealant group (HR 0.33; 0.20-0.54). Caries increment was lower in toothbrushing children than in nontoothbrushing children. Hazard ratios reached statistical significance for the non-treated children (HR 0.43; 0.210.87 ) and the sealant-treated children (HR 0.15; 0.03 0.072).
The retention rate after 18 months was 58\% for complete and partially retained sealants and $42 \%$ for those that were totally missing. Retention rates differed between the toothbrushing and non-toothbrushing group but not statistically significantly.

\section{Discussion}

Practical considerations and unforeseen events that took place during the study meant that this study had several methodological limitations that unfortunately lower the level of evidence of the findings. Studies in developing countries have to deal with the prevailing realities where methodological considerations often have to succumb to political and resource demands. For instance, the selection of participating schools, six in Cagayan de Oro and two in Manila were from a methodological point of view less than optimal. However, this resulted from an insistence of the Central Office of the Department of Education (DepEd) that the study be undertaken not only in the city of Cagayan de Oro but also in the capital city of Manila. The distances incurred between the study locations therefore obliged a greater number of examiners than would be ideal. For this reason it was not practical to undertake inter-examiner's reproducibility in any meaningful way. Another methodological problem was the assignment of non-treatment (control) children. The intention was to randomize the first graders of the eight schools into those who received treatment and those who received no treatment. Since the treatment was provided on the school premises, the principals and local educational officers did not authorize a design where certain children in the same school would serve as nontreatment controls. Separate schools therefore had to be used for the non-treatment controls.

Randomized controlled study designs have become the 'gold standard' in research [26]. However, it has been argued that the application of such high quality study design is often logistically impossible and unaffordable owing to limited recourses [27]. Researchers are often confronted with such difficulties when conducting community-based research in countries with limited resources. Nevertheless, we believe that conducting clinical research in countries where oral health problems are high is essential since it is not always appropriate or

Table 4 Number of sound surfaces, number of surfaces with new dentinal caries (D3) lesions and caries increment after 18 months according to group

\begin{tabular}{|c|c|c|c|c|c|c|}
\hline & \multicolumn{3}{|c|}{ Brushing } & \multicolumn{3}{|c|}{ Non-brushing } \\
\hline & $\begin{array}{l}\text { Non-treated } \\
\mathrm{N}=127\end{array}$ & $\begin{array}{l}\text { SDF treated } \\
n=288\end{array}$ & $\begin{array}{l}\text { Sealants } \\
\mathrm{n}=301\end{array}$ & $\begin{array}{l}\text { Non-treated } \\
\mathrm{n}=544\end{array}$ & $\begin{array}{l}\text { SDF treated } \\
n=418\end{array}$ & $\begin{array}{l}\text { Sealants } \\
n=467\end{array}$ \\
\hline Sound (n) & 117 & 262 & 298 & 453 & 366 & 438 \\
\hline $\begin{array}{l}\text { New dentinal } \\
\text { (D3) caries (n) }\end{array}$ & 10 & 26 & 3 & 91 & 52 & 29 \\
\hline Caries increment & 0.08 & 0.09 & 0.01 & 0.17 & 0.12 & 0.06 \\
\hline
\end{tabular}


Table 5 Hazard ratios $(95 \% \mathrm{Cl})$ and p-values between treatment and non-treatment in brushing and non-brushing schools and between brushing and non-brushing schools

\begin{tabular}{lll}
\hline Groups comparison $(\mathbf{n}=$ surfaces) & Hazard ratio $\mathbf{( 9 5 \%} \mathbf{C l})$ and $\mathbf{p}$-value \\
\hline Brushing schools & & $1.16(0.51-2.63) p=0.72$ \\
\hline SDF treated $(n=288)$ & versus non-treated $(n=127)$ & $0.12(0.02-0.61) p<0.01$ \\
\hline Sealants $(n=301)$ & versus non-treated $(n=127)$ & $0.71(0.45-1.11) p=0.12$ \\
\hline Non-brushing schools & & $0.33(0.20-0.54) p<0.001$ \\
\hline SDF treated $(n=418)$ & versus non-treated $(n=544)$ & \\
\hline Sealants $(n=467)$ & versus non-treated $(n=544)$ & $0.43(0.21-0.87) p<0.02$ \\
\hline Brushing & versus non-brushing & $0.70(0.38-1.27) p=0.23$ \\
\hline Non-treated brushing $(n=127)$ & versus non-treated non-brushing $(n=544)$ & $0.15(0.03-0.72) p<0.02$ \\
\hline SDF treated brushing $(n=288)$ & versus SDF treated non-brushing $(n=418)$ & \\
\hline Sealants brushing $(n=298)$ & versus sealants non-brushing $(n=438)$ & \\
\hline
\end{tabular}

relevant to transfer study findings from developed countries to developing countries. It has been questioned if randomized controlled trials conducted under optimal conditions are able to address questions of effectiveness and efficiency of clinical interventions in health care systems adequately [27], because of the problems of applicability and transferability from controlled trial conditions to real-life conditions in communities.

The current study of 18 months duration, although with limitations, has already shown important findings. It would, however, be unwise to speculate on the longerterm results from the different groups.

The affluent upper and middle class in the Philippines educate their children in private schools. The remaining Filipino child population (about 90\%) attend public elementary schools because their parents cannot afford the school fees of private schools. Differences in oral health of these children between rural and urban areas are not apparent and all tend to have comparable socioeconomic backgrounds [4]. Nevertheless, differences in caries levels were seen at baseline between groups. The existing bias at baseline was, however, to a certain extent bypassed by analyzing caries increment over the 18months period.

The high mobility of people in the Philippines and frequent school absenteeism due to illness result in large dropout rates of children in elementary schools. The loss to follow-up rate in this study was in line with the official dropout rates in rural Mindanao, which are known to be the highest between grade one and grade two. This has to be taken into account when clinical research is planned.

The unforeseen lack of compliance with daily school toothbrushing in three of the study schools is an example of the difficulties encountered in such studies, but it allowed for the opportunity to conduct a further evaluation on the background effect of lack of brushing with fluoride toothpaste on the applied treatment regimes. The consequence of dividing the whole sample impacts negatively on the power of this study and unfortunately limits the interpretation of data.

A two-year school-based daily fluoride toothpaste brushing program in five-year-old school children in Scotland with high caries risk showed a $56 \%$ reduction in caries increment of the permanent first molars [28]. In Indonesia, a three-year school-based toothbrushing program with fluoride toothpaste in elementary schools resulted in up to a $40 \%$ reduction in caries [29]. The hazard ratio of 0.43 (CI; 0.21-0.87) for new caries development in the brushing groups with fluoride toothpaste is therefore consistent with results achieved from other school-based toothbrushing programs with fluoride toothpaste in deprived communities.

The sealants that were applied in the present study could be considered as both preventive sealants (on surfaces clinically free of caries) and therapeutic sealants on surfaces with all stages of enamel caries lesions up to but not including visible dentinal caries. Unfortunately, despite attempts to do so, it was impossible to distinguish between sound and the different stages of enamel caries lesions that precede dentinal caries in the present study since these stages of caries could not reliably be diagnosed in the described field setting in the absence of compressed air, suction and proper lighting.

The retention rates of ART sealants in this study were moderate. Other studies have reported retention rates of high viscosity GIC sealants that vary considerably [30-33]. This variance may be explained by the clinical conditions (field setting) under which sealants are placed, the teeth selected for sealing, operator factors and inadequate training.

This study assessed the effect of SDF on preventing dentinal caries lesions but it was not designed to determine the degree of arrested caries that has been reported in other controlled clinical trials [18,22]. Only one controlled clinical trial in Cuba on 38\% SDF application for the prevention of new caries (D3) on occlusal surfaces of permanent first molars has been published [18]. After 
three years of biannual application of SDF, a significant caries reduction of $64 \%$ was reported, but the results are difficult to interpret, since most of the occlusal surfaces in the control group had received restorations. The present study is the first clinical trial that assesses the caries preventive efficacy of SDF application in children who brushed daily at school with fluoride toothpaste and is the second study after that of Yee et al. [22] that evaluates the effect of a single application of $38 \%$ SDF. In common with this last study, tannic acid was used as an inexpensive reducing agent after SDF application to accelerate the deposition of silver phosphate [34]. Recently, it has been shown that this extra step might not be necessary, since tannic acid did not appear to have any additional effect [22]. The present study showed no additional caries preventive effect, 18 months after a single application of $38 \%$ SDF on permanent first molars of six- to eight-year-old children who brushed their teeth daily at school with fluoride toothpaste. It is also questionable whether SDF has any efficacy in preventing new caries in children who are not under a toothbrushing with fluoride toothpaste regime. The present finding urges more research on the efficacy of SDF on the onset of new caries.

\section{Conclusions}

In this field study, conducted in Filipino elementary schools, a one-time application of $38 \%$ SDF on the occlusal surfaces of permanent first molars of six- to eightyear-old children was not an effective method to prevent the onset of new dentinal (D3) caries lesions in children. ART sealants significantly reduced the onset of new dentinal (D3) caries lesions over a period of 18 months.

\section{Competing interests}

The authors declare that they have no competing interests.

\section{Authors' contributions}

BM was the principal investigator and prepared the first draft of the paper. JM performed the statistical data analysis, WVP contributed to the critical interpretation of the data. RHW and $\mathrm{CH}$ were involved in the data collection and interpretation. All authors contributed equally to the final version of the paper and have read as well as approved the final manuscript.

\section{Authors' information}

BM worked in the frame of the 'integrated expert program' of the German Development Cooperation as advisor to the Department of Education and supported the Department in the development of low-cost strategies to improve oral health of children in the Philippines.

\footnotetext{
Acknowledgements

The authors would like to thank the health personnel, administrators and teachers of the Department of Education in the Philippines who participated and supported this research. Special thanks must be given to Dr Jun Araojo, Chief Dentist of the Department of Education, who organized the survey and took care of the logistics and Dr Robert Yee who participated in the data gathering. In addition, the authors acknowledge the support of $3 \mathrm{M}$ ESPE for supplying the glass-ionomer material used in the study and the Centre for International Migration and Development (CIM)/Gesellschaft für Internationale Zusammenarbeit (GIZ) GmbH for financing other consumable materials and travel.
}

The first author conducted this study as an employee of the Department of Education, financially supported by GIZ.

None of the authors received any funding for writing the paper.

\section{Author details}

${ }^{1}$ Deutsche Gesellschaft für Internationale Zusammenarbeit (GIZ) GmbH, GIZ Office Manila, PDCP Bank Centre, V.A. Rufino cor. L.P. Leviste Str, Makati City, Metro Manila, Philippines. ${ }^{2}$ Department of Preventive and Paediatric Dentistry, Jena University Hospital, WHO Collaborating Centre for Prevention of Oral Diseases, Bachstr. 18, 07743, Jena, Germany. ${ }^{3}$ Department of Global Oral Health, College of Dental Sciences, Radboud University Nijmegen Medical Centre, 6500 HB, Nijmegen, The Netherlands. ${ }^{4}$ Aide Odontologique Internationale, 1 Rue Maurice Arnoux, 92120, Montrouge, France. ${ }^{5}$ Dental Health International Nederland (DHIN), Korte Linschoten OZ 14, 3461 CG, Linschoten, The Netherlands.

Received: 11 November 2011 Accepted: 31 October 2012 Published: 21 November 2012

\section{References}

1. Edelstein BL: The dental caries pandemic and disparities problem. BMC Oral Health 2006, 1(Suppl):S2.

2. Petersen PE, Bourgeois D, Ogawa H, Estupinan-Day S, Ndiaye C: The global burden of oral diseases and risks to oral health. Bull World Health Organ 2005, 83:661-669.

3. Baelum V, Van Palenstein Helderman WH, Hugoson A, Yee R, Fejerskov O: A global perspective on changes in the burden of caries and periodontitis: implications for dentistry. J Oral Rehabil 2007, 34:872-906.

4. Monse B, Benzian H, Holmgren C, van Palenstein Helderman W, HeinrichWeltzien R: A silent public health crisis: Untreated caries and dental infections among 6- and 12-yr-old children in the Philippine National Oral Health Survey 2006. Asia Pac J Publ Health 2006, in press.

5. Monse B, Heinrich-Weltzien R, Benzian H, Holmgren C, van Palenstein Helderman WH: PUFA - An index of clinical consequences of untreated dental caries. Community Dent Oral Epidemiol 2010, 38:77-82.

6. Araojo JR: Philippines country report: school oral health promotion programme. In Proceedings of the 2nd Asian conference on oral health promotion for school children. Prospectus for our future generation. Ayyuthaya Bangkok: Thammasat University; 2003:103-110.

7. Belizario V, de Leon WU, Lumampao YF, Anastacio MB, Tai CM: Sentinel surveillance of soil-transmitted helminthiasis in selected local government units in the Philippines. Asia Pacific J Publ Health 2009, 21:26-42.

8. Benzian H, Monse B, Heinrich-Weltzien R, Mulder J, Hobdell M, van Palenstein Helderman WH: Untreated severe dental decay: a neglected determinant of low Body Mass Index in 12-year-old Filipino children. BMC Publ Health 2011, 11:558.

9. Bratthall D, Hänsel Petersson G, Sundberg $\mathrm{H}$ : Reason for the caries decline: what do the experts believe? Eur J Oral Sci 1994, 104:416-422.

10. Marthaler TM, O'Mullane D, Vrbic V: The prevalence of dental caries in Europe 1990-95. Caries Res 1996, 30:237-255.

11. Frencken JE, Holmgren CJ, van Palenstein Helderman WH: Basic Package of Oral Care (BPOC). Nijmegen: WHO Collaborating Centre for Oral Health Care Planning and Future Scenarios. University of Nijmegen: College of Dental Science Radboud; 2002.

12. World Health Organization, FDI World Dental Federation, International Association for Dental Research: Call to action to promote dental health by using fluoride: Global Consultation on Oral Health through Fluorides. http:// www.who.int/oral_health/events/oral\%20healthc.pdf.

13. World Health Organization: World Health Assembly: Oral health: Action plan for promotion and integrated disease prevention: WHA60/R17. Geneva: WHO; 2007.

14. World Health Organization (WHO), FDI World Dental Federation, International Association for Dental Research (IADR), and Chinese Stomatological Association (CSA): Bejing Declaration: Call to action to promote oral health by using fluoride in China and Southeast Asia. Conference on Dental Health through Fluoride in China and Southeast Asia: 18-19 September 2007. Beijing, China: WHO, FDI, IADR, CSA; 2007.

15. Marinho VC, Higgins JP, Sheiham A, Logan S: Fluoride toothpastes for preventing dental caries in children and adolescents. Cochrane Database Syst Rev 2005, (3). doi:10.1002/14651858. CD002278. 
16. Carvalho JC, Ekstrand KR, Thylstrup A: Dental plaque and caries on occlusal surfaces of first permanent molars in relation to stage of eruption. J Dent Res 1989, 68:773-779.

17. Marinho VC, Higgins JP, Sheiham A, Logan S: Combinations of topical fluoride (toothpastes, mouthrinses, gels, varnishes) versus single topical fluoride for preventing dental caries in children and adolescents. Cochrane Database Syst Rev 2005, (3). doi:10.1002/14651858.CD002781.

18. Llodra JC, Rodríguez A, Ferrer B, Menardia V, Ramos T, Morato M: Efficacy of silver diamine fluoride for caries reduction in primary teeth and first permanent molars of schoolchildren: 36-month clinical trial. J Dent Res 2005, 84:721-724.

19. Rosenblatt A, Stamford TC, Niederman R: Silver diamine fluoride: a caries "silver-fluoride bullet". J Dent Res 2009, 88:116-125.

20. de Amorism RG, Leal SC, Frencken JE: Survival of atraumatic restorative treatment (ART) sealants and restorations: a meta-analysis. Clin Oral Investig 2012, 16:429-441

21. World Health Organization: Oral Health Surveys: Basic methods. 4th edition. Geneva: WHO; 1997.

22. Yee R, Holmgren C, Mulder J, Lama D, Walker D, van Palenstein Helderman W: Efficacy of silver diamine fluoride for arresting caries treatment. J Dent Res 2009, 88:644-647.

23. Frencken JE, Holmgren CJ: Atraumatic restorative treatment for dental caries. Nijmegen: STl; 1999.

24. Kalbfleisch JD, Prentice R: The statistical analysis of failure time data. Hoboken: John Wiley \& Sons; 1980.

25. Andersen PK, Klein JP, Knudsen KM, Tabanera y Palacios R: Estimation of variance in Cox's regression model with shared gamma frailties. Biometrics 1997, 53:1475-1484.

26. Starfield B: Quality-of-care research: Internal elegance and external relevance. J Am Med Assoc 1998, 280:1006-1008.

27. Wang S, Moss JR, Hiller JE: Applicability and transferability of interventions in evidence-based public health. Health Promot Int 2005, 21:76-83.

28. Curnow MMT, Pine CM, Burnside G, Nicholson JA, Chesters RK, Huntington E: A randomised controlled trial of the efficacy of supervised tooth brushing in high-caries-risk children. Caries Res 2002, 36:294-300.

29. Adyatmaka A, Sutopo U, Carlsson P, Bratthall D, Pakhomov G: School-based primary preventive programme for children. Affordable toothpaste as a component in primary oral health care. Experiences from a field trial in Kalimantan Barat, Indonesia. Geneva: WHO; 1998.

30. Ho TFT, Smales RJ, Fang DKS: A 2-year clinical study of two glass-ionomer cements used in the atraumatic restorative treatment (ART) technique. Community Dent Oral Epidemiol 1999, 27:195-201.

31. Holmgren CJ, Lo ECM, Hu DY, Wan H: ART restorations and sealants placed in Chinese school children - results after three years. Community Dent Oral Epidemiol 2000, 28:314-320.

32. Beiruti N, Frencken JE, van't Hof MA, Taifour D, van Palenstein Helderman WH: Caries preventive effect of a one-time application of composite resin and glass ionomer sealants after 5 years. Caries Res 2006, 40:52-59.

33. Kemoli AM, Opinya GN, van Amerongen WE: Two-year survival of glass ionomer sealants placed as part of proximal ART restorations. East Afr Med J 2010, 87:375-381.

34. Craig GC, Powell KR, Cooper MH: Caries progression in primary molars: 24-month results from a minimal treatment programme. Community Dent Oral Epidemiol 1981, 9:260-265.

doi:10.1186/1472-6831-12-52

Cite this article as: Monse et al:: Caries preventive efficacy of silver diammine fluoride (SDF) and ART sealants in a school-based daily fluoride toothbrushing program in the Philippines. BMC Oral Health 2012 12:52.

\section{Submit your next manuscript to BioMed Central and take full advantage of:}

- Convenient online submission

- Thorough peer review

- No space constraints or color figure charges

- Immediate publication on acceptance

- Inclusion in PubMed, CAS, Scopus and Google Scholar

- Research which is freely available for redistribution 